\title{
Structural Diversity of the Extensor Digitorum Profundus Muscle Complex in Platyrrhini
}

\author{
Mellin A. Novikova ${ }^{a} \quad$ Aleksandra A. Panyutina $^{b}$

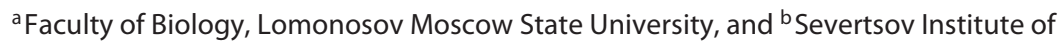 \\ Ecology and Evolution, Moscow, Russia
}

\begin{abstract}
Keywords
Platyrrhines · Muscles · Myology · Extensor of fingers · Musculus extensor digitorum profundus
\end{abstract}

\begin{abstract}
Separate extension of fingers in the hand of primates is performed by 3 muscles: $\mathrm{m}$. extensor pollicis longus, $\mathrm{m}$. extensor digiti secundi, and $\mathrm{m}$. extensor digitorum lateralis. Here it is proposed to consider them as parts of the extensor digitorum profundus muscular complex. The diversity in structure of these muscles in primates is examined based both on original anatomical study of New World monkeys and analysis of extensive published data on primates from different taxonomic groups. It is shown that in these muscles there are 2 main types of structure variations - the division of the muscle belly into several heads which give rise to separate tendons, and the split of the single terminal tendon into several branches. The first type of modification ensures the possibility of a separate management of the fingers, and the second, on the contrary, ensures the coupled control of extension of fingers. A scheme of evolutionary transformations of muscles belonging to the complex of the deep extensors of fingers is proposed.
\end{abstract}

๑ 2017 S. Karger AG, Basel

\section{Introduction}

New World primates form a well-defined monophyletic group, the Platyrrhini [Schneider and Sampaio, 2015]. The controversial phylogenetic relationships within this clade are not the issue of the present study, and we follow here the most supported classification, according to which platyrrhines are divided into 3 families: Pitheciidae is the most basal family, and the crown group includes the sister taxa Atelidae and Cebidae [Perelman et al., 2011; Springer et al., 2012]. All New World primates are

KARGER $\quad$ @ 2017 S. Karger AG, Basel
$\begin{aligned} & \text { E-Mail karger@karger.com } \\ & \text { www.karger.com/fpr }\end{aligned}$
Aleksandra A. Panyutina
Severtsov Institute of Ecology and Evolution
Vavilova Street 34, 102a
RU-119071 Moscow (Russia)
E-Mail myotis@ mail.ru 
mostly arboreal but differ in modes of locomotion and preferred ways to travel through the 3-dimensional space of the forest canopy [Hershkovitz, 1977]. They can use quadrupedal running and walking on horizontal and inclined branches (Callitrichinae, Saimiri, Aotus, Cebus, Cacajao), clinging to the vertical trunks and climbing on them (Callitrichinae), suspension and brachiation (Alouatta, Ateles, Lagothrix, Brachyteles), and leaping (Callitrichinae, Saimiri, Aotus, Cebus, Cacajao). Depending on the features of substrate and method of locomotion, different types of grasping are used: (1) manual grasp with lack of pollical opposition (usually with all 5 digits turned sideways relative to the support and somewhat flexed); (2) claw grasp on a vertical trunk (with the digits spread apart and aided by palm pads for fixing); (3) prehension of a branch opposing the thumb; (4) prehension of a branch spreading apart digits II and III to form a broad manual grasp (schizodactyly); (5) clinging to the branches with the digits II-V (but not the thumb) flexed as a hook [Youlatos, 1999, 2000].

The variety of grasping types in platyrrhine locomotion is reflected by a corresponding diversity of external morphological features of the manus (shape, size, palm skin texture, presence of claws or nails, etc.). The following manual morphological variations specific to the New World monkeys can be noted: (1) syndactyly or partial fusion of the fingers II-IV (Leontopithecus); (2) partial opposition of the thumb (Cebus); (3) "hook-like" manus with reduced thumb (Ateles); (4) schizodactyly, or spreading apart digits II and III to form a broad manus grasp (Alouatta, Cacajao); (5) absence of thumb opposition and presence of secondary claws (Callitrichinae); (6) strong development of the palmar and finger pads (Saimiri, Aotus, and to a lesser extent Callitrichinae) [Pocock, 1917; Napier, 1961; Ankel-Simmons, 2007].

With such a variety of grasping types affecting the external morphology of the hand, one might suppose a corresponding diversity in the anatomy of the forelimb. Nevertheless, the general structural plan of the skeleton and muscles of the forearm and hand in all monkeys (including catarrhines) looks very uniform [Dunlap et al., 1985]. This is not surprising, because the conservatism in the set and overall structure of limb muscles is not unique to primates, but is typical for the majority of quadrupedal mammals, save those whose limbs have undergone a strong transformation due to specialization.

However, there is no sufficient reason to abandon the assumption that the various locomotor specializations impose at least a faint mark on the structure of the limb muscles. The forelimb of platyrrhines was not deprived of anatomists' attention [Beattie, 1927; Robertson, 1944; Hill, 1957, 1959, 1960; Jouffroy and Lessertisseur, 1960; Hill, 1962; Grand, 1968; Schön, 1968; Bodini, 1981; Turnquist, 1983; Dunlap et al., 1985; Aversi-Ferreira et al., 2010; Diogo and Wood, 2012]. However, many species are poorly described morphologically. For instance, we failed to find any description of the musculoskeletal system of Cacajao and Leontopithecus in the literature, and descriptions of some other taxa of New World monkeys are sometimes very fragmentary and not always accompanied by detailed illustrations. To date the most comprehensive review of primate muscles (22 species including humans) in comparison with other mammals is presented in the fundamental survey of Rui Diogo and Bernard Wood [2012].

Among the most variable forearm muscles are the deep extensors of digits. According to many researchers [e.g., Aziz and Dunlap, 1986; Youlatos, 1999], evolutionary changes of the deep extensors of digits have a functional basis, i.e., they are associated with locomotor and manipulative modes. This is obvious, since only these muscles can provide an independent extension of fingers.

Extensor Digitorum Profundus Muscle Complex in Platyrrhini
Folia Primatol 2017;88:274-292 DOI: $10.1159 / 000478524$ 
Table 1. Nomenclature of long extensors of digits of the deep layer according to various authors: generalizing and splitting names

\begin{tabular}{|c|c|c|c|c|c|c|}
\hline George, 1977 & $\begin{array}{l}\text { m. abductor } \\
\text { pollicis longus }\end{array}$ & \multicolumn{3}{|c|}{ m. extensor digitorum radialis } & \multicolumn{2}{|c|}{ m. extensor digitorum ulnaris } \\
\hline \multirow{2}{*}{$\begin{array}{l}\text { Dunlap et al., 1985; } \\
\text { Aziz and Dunlap, 1986; } \\
\text { Hill, } 1953\end{array}$} & \multicolumn{4}{|c|}{ m. extensor digitorum profundus complex } & \multirow{2}{*}{\multicolumn{2}{|c|}{$\begin{array}{l}\text { m. extensor digiti quinti } \\
\text { et quarti proprius }\end{array}$}} \\
\hline & $\begin{array}{l}\text { m. abductor } \\
\text { pollicis longus }\end{array}$ & \multicolumn{3}{|c|}{ m. extensor digitorum profundus (proprius) } & & \\
\hline Youlatos, 1999, 2000 & $\begin{array}{c}\text { m. abductor } \\
\text { pollicis longus }\end{array}$ & \multicolumn{2}{|c|}{$\begin{array}{c}\text { m. extensor } \\
\text { indicis et pollicis longus }\end{array}$} & $\begin{array}{c}\text { m. extensor digiti } \\
\text { tertii proprius }\end{array}$ & \multicolumn{2}{|c|}{$\begin{array}{l}\text { m. extensor digiti IV } \\
\text { et } V \text { proprius }\end{array}$} \\
\hline Schön, 1968 & $\begin{array}{l}\text { m. abductor } \\
\text { pollicis longus }\end{array}$ & \multicolumn{2}{|c|}{ m. extensor pollicis et indicis longus } & $\begin{array}{l}\text { m. extensor digiti } \\
\text { tertii proprius }\end{array}$ & \multicolumn{2}{|c|}{$\begin{array}{l}\text { m. extensor digiti quinti } \\
\text { et quarti proprius }\end{array}$} \\
\hline Turnquist, 1983 & $\begin{array}{l}\text { m. abductor } \\
\text { pollicis longus }\end{array}$ & - & \multicolumn{2}{|c|}{$\begin{array}{l}\text { m. extensor digitorum indicis et } \\
\text { tertii proprius }\end{array}$} & \multicolumn{2}{|c|}{$\begin{array}{l}\text { m. extensor digiti quinti } \\
\text { (et quarti) proprius }\end{array}$} \\
\hline \multirow{2}{*}{ Duckworth, 1904} & \multirow{2}{*}{$\begin{array}{l}\text { m. extensor } \\
\text { ossis metacarpi } \\
\text { pollicis }\end{array}$} & \multirow{2}{*}{$\begin{array}{l}\text { m. extensor primi } \\
\text { (secundi?) } \\
\text { internodii pollicis }\end{array}$} & \multirow{2}{*}{$\begin{array}{l}\text { m. extensor } \\
\quad \text { indicis }\end{array}$} & $\begin{array}{l}\text { m. extensor digiti } \\
\text { medii }\end{array}$ & \multicolumn{2}{|c|}{ m. extensor digiti minimi } \\
\hline & & & & \multicolumn{2}{|c|}{$\begin{array}{c}\text { m. extensor digiti medii et digiti } \\
\text { annularis }\end{array}$} & $\begin{array}{c}\text { m. extensor } \\
\text { digiti minimi }\end{array}$ \\
\hline Danilova, 1979 & $\begin{array}{l}\text { m. abductor } \\
\text { pollicis longus }\end{array}$ & $\begin{array}{l}\text { m. extensor } \\
\text { pollicis longus }\end{array}$ & $\begin{array}{c}\text { m. extensor } \\
\text { indicis proprius }\end{array}$ & - & \multicolumn{2}{|c|}{ m. extensor digiti minimi } \\
\hline Sinelnikov, 1989 & $\begin{array}{l}\text { m. abductor } \\
\text { pollicis longus }\end{array}$ & $\begin{array}{c}\text { m. extensor } \\
\text { pollicis longus }\end{array}$ & $\begin{array}{l}\text { m. extensor } \\
\text { indicis }\end{array}$ & - & - & $\begin{array}{l}\text { m. extensor } \\
\text { digitorum } \\
\text { minimi }\end{array}$ \\
\hline Panyutina et al., 2015 & $\begin{array}{l}\text { m. abductor } \\
\text { pollicis longus }\end{array}$ & $\begin{array}{l}\text { m. extensor } \\
\text { pollicis longus }\end{array}$ & \multicolumn{2}{|c|}{ m. extensor indicis } & \multicolumn{2}{|c|}{ m. extensor digitorum lateralis } \\
\hline Diogo and Wood, 2012 & $\begin{array}{l}\text { m. abductor pollicis } \\
\text { longus }\end{array}$ & $\begin{array}{l}\text { m. extensor pollicis } \\
\text { longus }\end{array}$ & \multicolumn{2}{|c|}{ m. extensor indicis } & $\begin{array}{l}\text { m. extensor } \\
\text { digiti quarti }\end{array}$ & $\begin{array}{l}\text { m. extensor } \\
\text { digiti minimi }\end{array}$ \\
\hline \multirow[b]{2}{*}{ Accepted here } & & \multicolumn{5}{|c|}{ extensor digitorum profundus complex } \\
\hline & $\begin{array}{l}\text { m. abductor pollicis } \\
\text { longus }\end{array}$ & $\begin{array}{l}\text { m. extensor pollicis } \\
\text { longus }\end{array}$ & m. extensor & digiti secundi & m. extensor & itorum lateralis \\
\hline
\end{tabular}

Classification of the extensors which can be attributed to the deep layer of the extensor muscles of digits is somewhat confusing (Table 1). In the typical case, $\mathrm{m}$. extensor pollicis longus provides tendons for digit I, m. extensor digiti secundi for digit II, and $\mathrm{m}$. extensor digitorum lateralis for digits IV-V (nomenclature according to Nomina anatomica veterinaria [International Committee on Veterinary Cross Anatomical Nomenclature, 2012]). However, in many mammals the extensors of the deep layer send their tendons, often crossing over each other, to a greater number of fingers, and that fact has a significant impact on the nomenclature used in their description. To describe the extensors of the deep layer, some authors used the generalized name m. extensor digitorum profundus or profundus complex [Hill, 1953; Dunlap et al., 1985; Aziz and Dunlap, 1986]. Another general term used is m. extensor digitorum brevis [Sullivan and Osgood, 1927] as opposed to m. extensor digitorum communis. If the muscular fascicles of that layer are separable over their entire length, they often receive special names according to the numbers or names of the digits which they control (e.g., m. extensor digiti tertii proprius).

From our point of view, the extensor digitorum profundus complex includes $\mathrm{m}$. extensor pollicis longus, $\mathrm{m}$. extensor digiti secundi, and $\mathrm{m}$. extensor digitorum lateralis. All these muscles are derived from the reptilian $\mathrm{mm}$. extensores digitorum breves [Diogo and Abdala, 2010] and are deep in their insertion and morphogenesis. 
The only muscle we consider superficial is $\mathrm{m}$. extensor digitorum communis, which not only originates superficially, but also extends to the manus most externally and always reaches the fingertips in mammals. There is no doubt about the assignment of $\mathrm{m}$. extensor pollicis longus and $\mathrm{m}$. extensor digiti secundi to a deep layer - these muscles originate on the forearm, and their bellies are fully covered by superficial muscles. The $\mathrm{m}$. extensor digitorum lateralis originates on the lateral epicondyle of the humerus, together with the m. extensor digitorum communis, and, based on their association, could be erroneously attributed to the same superficial layer as the latter. However, firstly, it enters the manus under the deepest loop of the retinaculum extensorum and not at all together with $\mathrm{m}$. extensor digitorum communis, which goes below the superficial loop of the retinaculum extensorum. Secondly, during ontogenesis $\mathrm{m}$. extensor digitorum lateralis starts to form "in depth" together with m. extensor digiti secundi [Čihák, 1991], i.e., its place in the deep layer is beyond doubt, and the origin on the humeral epicondyle is explained just by the need to optimally arrange muscular attachments in a limited space.

In contrast to Aziz and Dunlap [1986], we do not include m. abductor pollicis longus in the extensor digitorum profundus complex, as it does not belong to the digital extensors in the proper sense terminating on the metacarpal I base and prepollex, and originally, it is a supinator of the manus ( $\mathrm{m}$. supinator manus of lower tetrapods [Straus, 1941]). In humans there is an additional $\mathrm{m}$. extensor pollicis brevis derived from $\mathrm{m}$. abductor pollicis longus, which we do not include within the extensor digitorum profundus complex either.

The objectives of this study include a detailed description of the deep layer of the extensor muscles of the forearm, comparative anatomical analysis of these muscles in New World primates, as well as comparison of platyrrhines in this respect with other primates and certain members of different mammalian orders. We suppose that the degree of differentiation of these muscles may be crucial for fine control of the prehensive pattern sensu Bishop [1962], which is the preparatory manual gesture determining how the fingers are set before capturing an object. This is a novel direction of research based on the fact that the role of extensor muscles in grasping is generally underestimated. Indeed, the flexors-over-extensors prevalence is known to be considerably greater in climbers than in walkers and runners, and mechanical reasons for this fact are quite obvious [Sustaita et al., 2013]. The prevailing role of flexors in the prehensive grip is indispensable, but weak extensors may be more important in the preparatory prehensive pattern. Here we consider the structural basis for separate extension of digits based on research of extensor muscular supply in platyrrhines, known for variety of grasping specializations. So, our purpose is morphofunctional, not phylogenetic.

\section{Materials and Methods}

Dissection of the forearm and manus muscles was undertaken in 9 platyrrhine species from all 3 families. Pitheciidae: bald uakari, Cacajao calvus (I. Geoffroy, 1847) (1 juvenile); Atelidae: Venezuelan red howler, Alouatta seniculus (Linnaeus, 1766) (1 juvenile); Cebidae: common marmoset, Callithrix jacchus (Linnaeus, 1758) (2 adults), black-tufted marmoset, Callithrix penicillata (E. Geoffroy, 1812) (1 adult), pygmy marmoset, Cebuella pygmaea (Spix, 1823) (1 adult), golden lion tamarin, Leontopithecus rosalia (Linnaeus, 1766) (1 adult), white-headed capuchin, Cebus capucinus (Linnaeus, 1758) (1 adult), tufted capuchin, Sapajus apella (Linnaeus, 1758)

Extensor Digitorum Profundus Muscle Complex in Platyrrhini
Folia Primatol 2017;88:274-292 DOI: $10.1159 / 000478524$ 


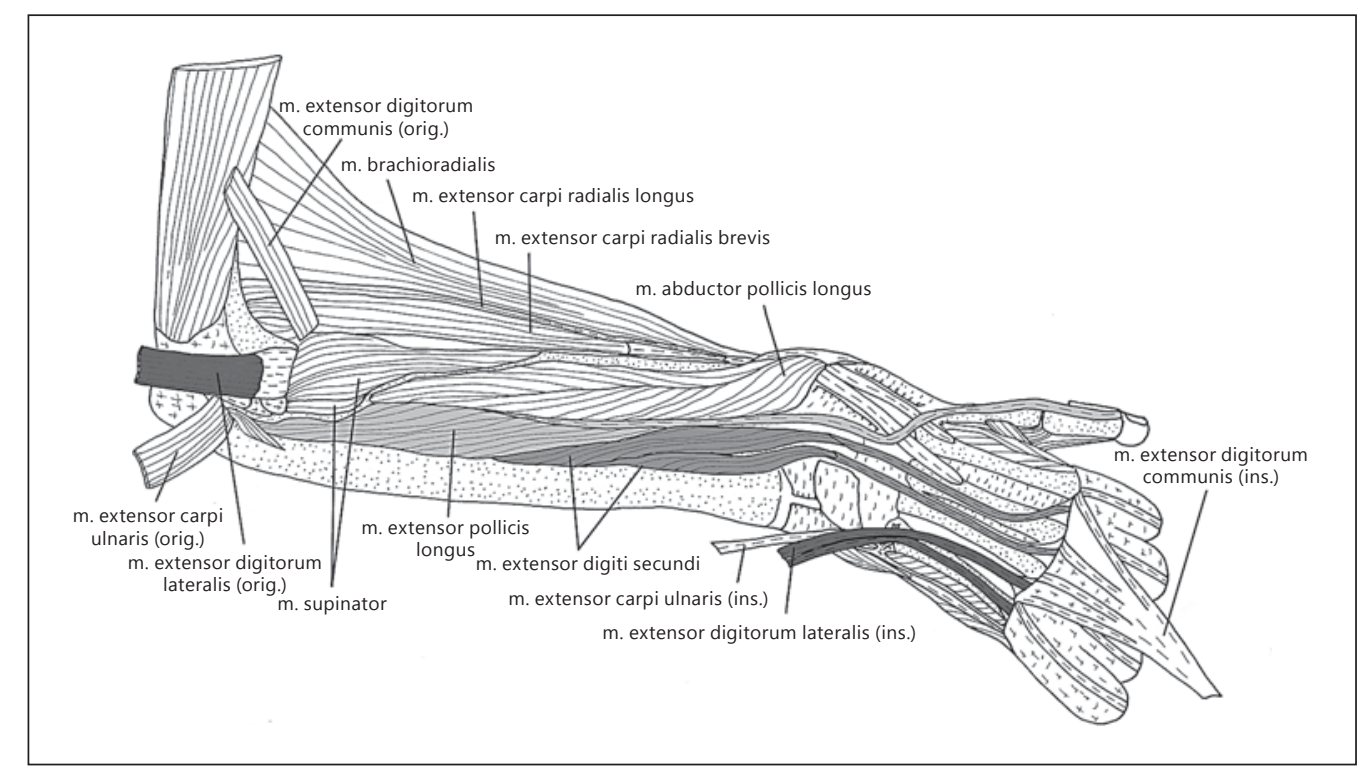

Fig. 1. Dorsal forearm musculature of Cacajao calvus; other muscles are not labelled. The second layer of dissection is shown: the superficial-most and some deeper forearm extensors are cut and turned aside.

(1 adult), common squirrel monkey, Saimiri sciureus (Linnaeus, 1758) (1 juvenile, 1 adult); and 1 catarrhine: green monkey, Chlorocebus sabaeus (Linnaeus, 1758) (1 juvenile). The material for dissection was provided by the Department of Vertebrate Zoology and the Zoological Museum of Moscow State University and the Zoological Institute of the Russian Academy of Sciences, Saint Petersburg. Anatomical procedures were performed using a stereomicroscope Carl Zeiss Stemi SV 11. All stages of dissection were filmed with a digital camera Canon PC1562. For each species, detailed descriptions of muscle topography, including the arrangement of fibres and tendons, and detailed anatomical drawings were made. Location and basic action of the muscles were assessed in situ during dissections. On the basis of photos and drawings, the schematic diagrams were generated, which helped to formalize functional classification of the observed structural types.

\section{Results}

In all the studied primates, the dorsal group of forearm musculature always consists of 10 muscles: $\mathrm{m}$. supinator, $\mathrm{m}$. brachioradialis, $\mathrm{m}$. extensor carpi radialis longus, $\mathrm{m}$. extensor carpi radialis brevis, $\mathrm{m}$. extensor digitorum communis, $\mathrm{m}$. extensor pollicis longus, $\mathrm{m}$. extensor digiti secundi, $\mathrm{m}$. extensor digitorum lateralis, $\mathrm{m}$. abductor pollicis longus, and $\mathrm{m}$. extensor carpi ulnaris (Fig. 1). Only in Alouatta was there found an eleventh abnormal muscle, which we described as $\mathrm{m}$. contrahens cubiti [Novikova and Kuznetsov, 2017]. We failed to find a separate m. epitrochleoanconeus lateralis (the homologue of reptilian and amphibian m extensor antebrachii ulnaris [Haines, 1939; Gambaryan et al., 2015]) in any dissected monkey. A detailed description of structural variations of 3 of them (m. extensor pollicis longus, 
$\mathrm{m}$. extensor digiti secundi, and $\mathrm{m}$. extensor digitorum lateralis), which form the extensor digitorum profundus complex, is provided in Table 2 .

An important distinctive feature of the muscles belonging to the extensor digitorum profundus complex in primates, as opposed to other mammals, is their insertion. In primates their terminal tendons fuse with respective tendons of m. extensor digitorum communis into a single tendinous sheath covering the dorsal side of the digit up to the nail base. So the deep extensors participate in extension of the distal interphalangeal joints together with $\mathrm{m}$. extensor digitorum communis. Contrary to that, in other mammals the deep extensor tendons pass under those of m. extensor digitorum communis separately and terminate on the pre-ungual phalanges.

\section{M. extensor pollicis longus}

In all studied specimens, the most medial muscle of the deep extensor complex is $\mathrm{m}$. extensor pollicis longus. This muscle usually originates side by side and distal to $\mathrm{m}$. abductor pollicis longus on the middle portion of the ulna, and on the interosseous membrane. The length of the initial aponeurosis and the position on the ulna relative to the adjacent extensors varies in different species. In most taxa it has 1 head, only in Cebus did we observe its division into 2 heads. The elongated muscle belly passes into a terminal tendon at the middle or distal part of the forearm.

In the platyrrhines dissected, a single terminal tendon at the distal part of the forearm or at the manus usually splits into 2, supplying digits I and II, and in Cebuella pygmaea, it splits into 3 - to digits I-III.

Although the set of finger tendons of $\mathrm{m}$. extensor pollicis longus varies in primates, in the case of a normally developed thumb, this muscle always sends a tendon to it. However, there are usually additional tendons to digit II or digits II and III. As the lateral portion, bearing tendons to the second (as well as to the third) digit, never replaces $\mathrm{m}$. extensor digiti secundi, there is every reason to believe that this lateral portion is the proper derivative of $\mathrm{m}$. extensor pollicis longus, and accordingly the entire muscle is a complete homologue of the human m. extensor pollicis longus, although the latter supplies the thumb only.

In the case of a reduced thumb, the muscle can be absent, as in Ateles geoffroyi [Turnquist, 1983], or insert on the vestigial skeletal elements of digit I, as in Hylobates [Straus, 1941], and may serve both the rudimentary thumb and digit II (in Ateles geoffroyi [Dunlap et al., 1985]), and in rare cases can lose connection with the thumb and serve only digit II [Aziz and Dunlap, 1986].

\section{M. extensor digiti secundi}

The second, more laterally lying muscle of the deep extensor complex is what we call $\mathrm{m}$. extensor digiti secundi. Its tendons, apart from digit II, can serve all the fingers, except the first. M. extensor digiti secundi is located in the deep layer of the dorsal forearm muscles.

In platyrrhines, $\mathrm{m}$. extensor digiti secundi originates from the ulna and (sometimes) from the adjacent part of the interosseous membrane of the forearm, distal to $\mathrm{m}$. extensor pollicis longus with which it often has a common initial aponeurosis. Because of this, some authors consider them a single muscle [Hill, 1953; Dunlap et al., 1985; Aziz and Dunlap, 1986]. In Leontopithecus these 2 muscles form a single muscular mass which gives tendons to digits I-IV [Dunlap et al., 1985]. Unfortunately, the original description does not allow one to determine the actual associa-

Extensor Digitorum Profundus Muscle Complex in Platyrrhini
Folia Primatol 2017;88:274-292 DOI: $10.1159 / 000478524$ 
Table 2. Variants of structure of the extensor digitorum profundus complex in studied primates

M. extensor digitorum lateralis (EDL)

Diagram
Cebuella pygmaea
lateral surface of the ulna, from the
interosseous membrane, and from the
fascia of the forearm

Origin: from the distal quarter of the lateral surface of the ulna The single muscle head reaches retinaculum extensorum, the common terminal tendon at the manus is divided into 2 - to digits II and IV

Insertion: the medial tendon of $\mathrm{m}$. extensor digiti secundi merges with the most lateral tendon of m. extensor pollicis longus to the third finger, and together they join the third finger tendon of m. extensor digitorum communis at its postaxial side; laterally it merges with tendon IV of $\mathrm{m}$. extensor digitorum lateralis which approaches from the postaxial side
Origin: from the lateral epicondyle of the humerus; it has a common initial aponeurosis with $\mathrm{m}$. extensor digitorum communis from which muscle fibres depart throughout the proximal two thirds or half of the forearm; this origin was observed in the majority of the species examined, so we consider it typical

One muscle head; the muscle belly

lies in the proximal half of the

forearm; the common termina tendon is divided into 2 at the wrist region

Insertion: At the manus both ten-

dons may be fused with tendons of

$\mathrm{m}$. extensor digiti secundi by thin aponeurotic ligaments; tendons approach digits IV and V from the postaxial side and join the tendons of $\mathrm{m}$. extensor digitorum communis abreast the proximal phalanges; such an insertion of the muscle was observed in the majority of the species examined, so we consider it typical

\section{Callithrix penicillato}

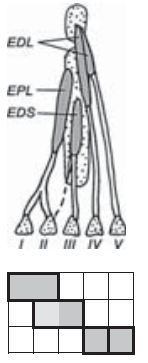

Origin: from the middle part of the lateral surface of the ulna, the interosseous membrane, and the fascia of the forearm

One muscle head; muscle fibres are short; a long terminal tendon passes on the wrist, where it thickens and branches into 2 equal tendons Insertion: one of the terminal tendons serves the thumb, the second one partly unites with a thin branch of the tendon of $\mathrm{m}$. extensor digiti secundi and passes to digit II; insertion of tendons is typical
Origin: from the third quarter of the lateral surface of the ulna

Two muscle heads turn into 2 tendons before reaching the retinaculum extensorum

Insertion: the thinner preaxial tendon passes to the postaxial tendon of $\mathrm{m}$. extensor pollicis longus and partially merges with it; serves digit to the third finger; attachment is typical, by merging with the terminal tendons of $\mathrm{m}$. extensor digitorum communis II; the thicker postaxial tendon goes
Callithrix jacchus

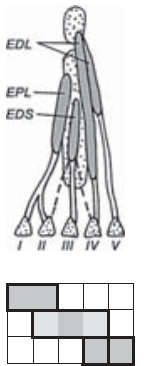

Origin: on a narrow sector along the ulnar edge of the proximal three quarters of the ulna

One muscle head

Insertion: as in Callithrix penicillata

1 terminal tendon is divided into 2

which are typically attached
Origin: from the distal quarter of the lateral surface of the ulna and a small section of the interosseous membrane

The muscle head turns into a single tendon before reaching the retinaculum extensorum

Insertion: the main tendon approaches the third finger from the postaxial side and merges with tendon III of $\mathrm{m}$. extensor digitorum communis; at the wrist area 2 thin ligaments branch off the main tendon and pass to digits II and IV; they represent a hardening of the deep backside aponeurosis
Origin: typical

Two heads lying in the proximal part of the forearm

Medial head turns into the terminal tendon in mid-forearm, and the lateral one - at the distal third of the forearm

Insertion: attachment of the terminal tendons is typical

Origin: typical

Two muscle heads; the postaxial head turns into the terminal tendon at the beginning of the distal third of the forearm; muscle fibres of the preaxial head almost reach the retinaculum extensorum

Insertion: attachment of the terminal tendons is typical 
Table 2 (continued)

\begin{tabular}{|c|c|c|c|}
\hline Diagram & M. extensor pollicis longus (EPL) & M. extensor digiti secundi (EDS) & M. extensor digitorum lateralis (EDL) \\
\hline \multicolumn{4}{|c|}{ Leontopithecus rosalia } \\
\hline EOL & $\begin{array}{l}\text { Origin: from the middle part of the } \\
\text { lateral surface of the ulna, the interosse- } \\
\text { ous membrane, and the fascia of the } \\
\text { forearm } \\
\text { One muscle head; it turns into a terminal } \\
\text { tendon at the middle of the forearm } \\
\text { Insertion: the terminal tendon bifurcates } \\
\text { at the level of the metacarpophalangeal } \\
\text { joints into branches to digits I and II }\end{array}$ & $\begin{array}{l}\text { Origin: distal third of the lateral } \\
\text { surface of the ulna } \\
\text { Two muscle heads with separate } \\
\text { tendons; the muscle fibres of the } \\
\text { lateral one reach the retinaculum } \\
\text { extensorum } \\
\text { Insertion: the terminal tendon of the } \\
\text { medial head runs to digit II and also } \\
\text { sends a minor branch to digit III; } \\
\text { and the terminal tendon of the lateral }\end{array}$ & $\begin{array}{l}\text { Origin: typical } \\
\text { Two muscle heads; the preaxial one } \\
\text { is very long, stretches all over the } \\
\text { forearm and nearly reaches the } \\
\text { retinaculum extensorum; the post- } \\
\text { axial one is much shorter and is } \\
\text { located in the proximal quarter of the } \\
\text { forearm } \\
\text { Insertion: the distal tendons join the } \\
\text { distal tendon of } m \text {. extensor digito- }\end{array}$ \\
\hline 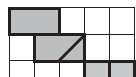 & & $\begin{array}{l}\text { head runs to digit III; attachment is } \\
\text { typical }\end{array}$ & $\begin{array}{l}\text { rum communis abreast the first } \\
\text { interphalangeal joints of fingers IV } \\
\text { and } V \text {, respectively }\end{array}$ \\
\hline
\end{tabular}

Saimiri sciureus

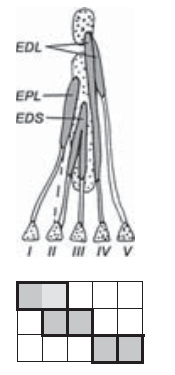

Origin: from the proximal third of the lateral surface of the ulna and from the fascia of the forearm

One muscle head

Insertion: long and thin terminal tendon to digit I in the distal quarter of the

forearm provides an additional very thin ligament to digit II, but it gets lost in the overall aponeurotic layer of deep extensors
Origin: from the distal half of the ulna

The muscle mass is subdivided into 2 weakly expressed heads that do no reach the retinaculum extensorum Insertion: 2 distal tendons to digits II and III; the tendon of digit II with its transfer to the wrist accepts a ligament from $\mathrm{m}$. extensor pollicis longus; attachment is typical
Origin: apart from the lateral epicondyle of the humerus, the muscle also originates from the radial head and the capsule of the radio-ulnar joint Two muscle heads; the preaxial one is very long, stretches all over the forearm and nearly reaches the retinaculum extensorum; the postaxial one is half the length and is located in the proximal half of the forearm

Insertion: attachment of the terminal tendons is typical

\section{Sapajus paella}

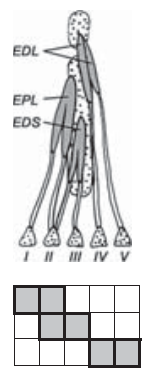

Cebus capucinus

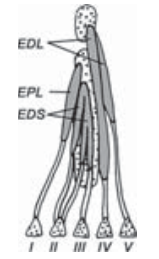

Origin: from the proximal third of the lateral surface of the ulna and from the fascia of the forearm

One muscle head; however, the muscle head giving 1 of the 2 tendons to the second finger may belong to $\mathrm{m}$. extensor pollicis longus and not to $\mathrm{m}$. extensor digitorum indicis

Insertion: the single terminal tendon supplies the thumb tendons which approach digits I and

from the preaxial side; attachment is

typical
Origin: from the third quarter of the ulna

Muscle fibres do not reach the retinaculum extensorum; in the distal quarter the muscle belly is partially divided Insertion: the terminal tendons pass to digits II and III, attachment is typical
Origin: typical

Two muscle heads lie in the proximal third of the forearm, wherein the few muscle fibres of the preaxial head accept a bunch of fibres from $m$. extensor digitorum communis Insertion: attachment of the terminal tendons is typical

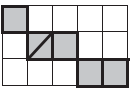

Origin: from the third quarter of the ulna

Muscle fibres do not reach the retinaculum extensorum; in the distal quarter the muscle belly is divided into three

Insertion: the terminal tendons pass to digits II (2 ligaments) and III, attachment is typical
Origin: typical

Two muscle heads; the preaxial one is very long, stretches all over the forearm and nearly reaches the retinaculum extensorum; the postaxial one is shorter and is located in the proximal three quarters of the forearm

Insertion: attachment of the terminal tendons is typical
Extensor Digitorum Profundus Muscle Complex in Platyrrhini
Folia Primatol 2017;88:274-292 
Table 2 (continued)

\begin{tabular}{|c|c|c|c|}
\hline Diagram & M. extensor pollicis longus (EPL) & M. extensor digiti secundi (EDS) & M. extensor digitorum lateralis (EDL) \\
\hline \multicolumn{4}{|l|}{ Cacajao calvus } \\
\hline EPL & $\begin{array}{l}\text { Origin: from the lateral surface of the } \\
\text { ulna in the area from the base of the } \\
\text { proximal epiphysis to the middle of the } \\
\text { forearm } \\
\text { One muscle head which covers a margin } \\
\text { of the m. abductor pollicis longus belly } \\
\text { Insertion: the single terminal tendon } \\
\text { supplies the thumb, attachment is typical }\end{array}$ & $\begin{array}{l}\text { Origin: from the third quarter of the } \\
\text { lateral surface of the ulna } \\
\text { Almost from the initial part the } \\
\text { muscle belly is divided into } 2 \text { heads } \\
\text { which reach the retinaculum exten- } \\
\text { sorum } \\
\text { Insertion: the terminal tendons } \\
\text { approach digits II and III from the } \\
\text { postaxial side, attachment is typical }\end{array}$ & $\begin{array}{l}\text { Origin: typical } \\
\text { Two muscle heads; the preaxial one } \\
\text { is longer and lies in the proximal two } \\
\text { thirds of the forearm; the postaxial } \\
\text { one is shorter and reaches about the } \\
\text { middle of the forearm } \\
\text { Insertion: attachment of the terminal } \\
\text { tendons is typical }\end{array}$ \\
\hline
\end{tabular}

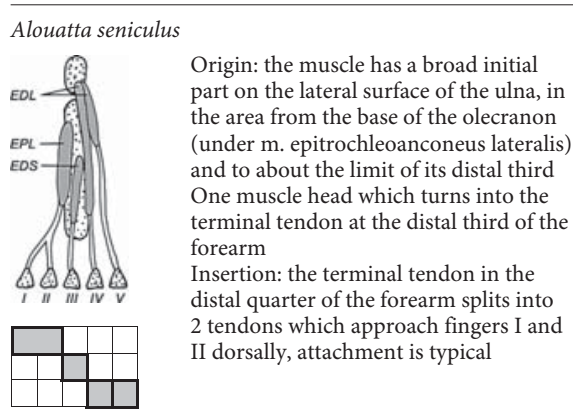

Origin: from the third quarter of the lateral surface of the ulna The single muscle head reaches the retinaculum extensorum Insertion: the single tendon serves digit III, attachment is typical
Origin: typical

Two muscle heads; the preaxial one is long and reaches the retinaculum extensorum; the postaxial one is shorter and is located in the proximal third of the forearm Insertion: attachment of the terminal tendons is typical

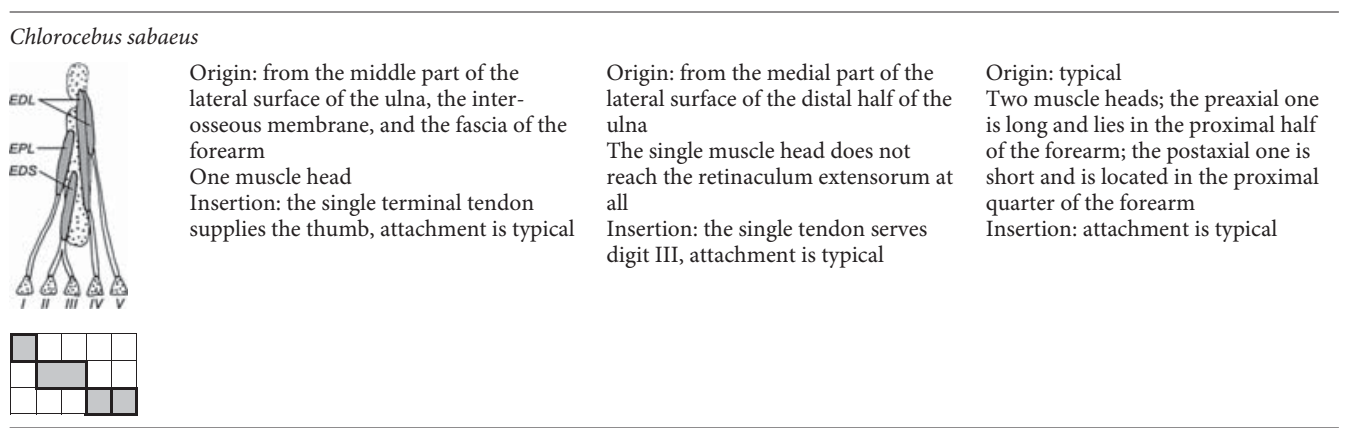

tions for each of the terminal tendons. In our specimen the muscle bellies were well separated, and the distribution of finger tendons was different from that specified by Dunlap et al. [1985].

Sometimes $\mathrm{m}$. extensor digiti secundi is also hard to separate from $\mathrm{m}$. abductor pollicis longus with which it can share the initial aponeurosis [Hill, 1957; Diogo and Wood, 2012]. Hill [1960] mentioned that in Cebus m. extensor digiti secundi originated over the entire forearm including the ulna, radius and the interosseous membrane, but this has not been confirmed by other researchers. In Tarsius [Burmeister, 1846] and Galago senegalensis [Stevens et al., 1977], an extra origin of m. extensor 
digiti secundi on the radius was described. In gibbons and orang-utans, an additional origin on the humerus was observed [Deniker, 1885], and in gorillas and chimpanzees, on the radius [Sonntag, 1923; Diogo and Wood, 2012]. In humans, m. extensor digiti secundi originates only on the ulna [Sinelnikov, 1989].

The number of heads of $\mathrm{m}$. extensor digiti secundi varies in primates. In Callithrix, Alouatta, and Chlorocebus, the muscle has 1 head which turns into a single terminal tendon just before the carpal bend. In Leontopithecus, Cebus, Saimiri, and Cacajao, muscle fibres pass into 2 separate terminal tendons (to digits II and III).

The distal tendons of the muscle at the metacarpus usually have more or less distinct connective tissue cross-links; however, they do not form such a firm integrating aponeurosis, as in $\mathrm{m}$. extensor digitorum communis. The terminal tendons above the basal phalanges join the corresponding tendons of m. extensor digitorum communis, typically approaching them from the lateral side.

As stated above, the number of finger tendons of $m$. extensor digiti secundi varies significantly. According to data from the literature summarized below, the most common variants for primates are: II-III and II-IV; moreover, additional variants have been described: I-III, II-V, II, III, III-IV, II and IV, II and V, III and V.

\section{Most Common Variants}

Variant II-III. According to our data this is the most typical variant for platyrrhines. We found it in almost all the investigated specimens. In many strepsirrhines, tarsiers, cercopithecids (except baboons), as well as in some hominoids, such as the orang-utan, the variant II-III also prevails [Burmeister, 1846; Woollard, 1925; Straus, 1941; Hill, 1953, 1957, 1960; Stevens et al., 1977; Turnquist, 1983; Aziz and Dunlap, 1986; Soligo, 2005; Diogo and Wood, 2012].

Variant II-IV. We have noted it only in Cebuella pygmaea (a single terminal tendon divides into 3 finger tendons at the metacarpus). According to the literature, among platyrrhines this variant is found in Callitrichinae [Bodini, 1985; Aziz and Dunlap, 1986; Aversi-Ferreira et al., 2010; Diogo and Wood, 2012], capuchins [Dunlap et al., 1985; Aversi-Ferreira et al., 2010], sakis [Ziemer, 1972, as cited in AversiFerreira et al., 2010; Diogo and Wood, 2012], and woolly monkeys [Robertson, 1944]. Furthermore, this variant also occurs in strepsirrhines, for instance in Daubentonia, and in tarsiers [Woollard, 1925; Soligo, 2005; Diogo and Wood, 2012]. In baboons the variant II-IV occurs as an exception [Kaneff, 1980, as cited in Diogo and Wood, 2012]; in gibbons it is the most common variant [Straus, 1941; Diogo and Wood, 2012].

Variant II. We have not found this variant in New World monkeys although it has been mentioned by Gremyatskiy [1933] for Callithrix jacchus. Among catarrhines, it is mentioned as an exception in Papio [Kaneff, 1980, as cited in Diogo and Wood, 2012]; it is the norm in Pan [Sonntag, 1923; Diogo and Wood, 2012] and Homo [Sinelnikov, 1989]. It is interesting to note that this variant is also found in $\mathrm{Tu}$ paia belangeri [Panyutina et al., 2015] and occurs as a variation in lorises, namely in Nycticebus [Hill, 1953; Diogo and Wood, 2012] and Loris [Murie and Mivart, 1872].

\section{Rare Variations}

Variant I-III. Recorded in only 1 specimen of Pongo [Diogo and Wood, 2012].

Variant II-V. It occurs as a variation in strepsirrhines and tarsiers [Diogo and Wood, 2012]. In addition, these authors also provide references to a number of sources indicating this variant in Hylobates and as a rare variation (5\%) in Pongo.

Extensor Digitorum Profundus Muscle Complex in Platyrrhini
Folia Primatol 2017;88:274-292 DOI: $10.1159 / 000478524$ 
Variant III. We have found it in Alouatta. The same pattern was observed in several howler specimens by Youlatos [1999]. It was listed for callitrichins, namely Saguinus [Straus, 1941; Hill, 1953].

Variant III-IV. We found this variant in only 1 specimen of Cebuella: the tendon was initially single, but then split on the back of the wrist into branches to digits III and IV. The same architecture of the terminal tendons was found in Callimico goeldii [Hill, 1959; Dunlap et al., 1985] and as an individual variation in Callicebus [Bodini, 1985]. In Callithrix jacchus, Beattie [1927] described a single tendon of this muscle supplying the third finger, but he also mentioned "a very thin slip" passing to digit IV $^{1}$. Diogo and Wood [2012] attributed this case to the variant II-IV. Additionally, variant III-IV was discovered in 3 species of Atelinae: Ateles geoffroyi (2 specimens), Ateles paniscus (1 specimen), and Lagothrix lagotricha (1 specimen) [Dunlap et al., 1985]. Among other primates, variant III-IV was found in Daubentonia [Soligo, 2005], with separate heads to these 2 digits.

Gorilla's Variant. The insertion of $\mathrm{m}$. extensor digiti secundi in gorilla is unusual. It is associated with digit II but, in addition, frequently shows various more proximal attachments, which include, in different combinations, the carpal bones capitatum and hamatum and the metacarpals III and IV [Diogo et al., 2010].

Most Rare Variants with "Missed Tendons"

Variant II and IV. It has been identified in Nycticebus [Barnard, 1876], and Pan [Straus, 1941].

Variant II and V. It has been identified in Nycticebus [Barnard, 1876].

Variant III and V. It has been recorded in Callicebus and Alouatta [Hill, 1953].

\section{M. extensor digitorum lateralis}

The complex of deep digital extensors also includes $\mathrm{m}$. extensor digitorum lateralis. It usually originates on the lateral epicondyle of the humerus, and in Saimiri also on the head of the radius and on the capsule of the elbow joint. In most taxa examined, the muscle belly, in its proximal third, is single but then divides into 2 heads (in $\mathrm{Ce}$ buella pygmaea it does not). The point where the muscle fibres pass into tendons and the splitting of the latter into several finger tendons vary in different species. In the manus, terminal tendons may be tied, by a thin connective aponeurosis, with the tendons of $\mathrm{m}$. extensor digiti secundi. The terminal tendons approach digits IV and V from the lateral side, and join the tendons of $\mathrm{m}$. extensor digitorum communis abreast the distal ends of the proximal phalanges (in Leontopithecus rosalia above the first interphalangeal joint); as part of the common extensor tendinous sheath they reach the bases of the ungual phalanges.

In tarsiers and most strepsirrhines, the $\mathrm{m}$. extensor digitorum lateralis operates 2 fingers (IV-V), but in lorises (Nycticebus and Loris), often only 1 (V) [Hill, 1953, 1960; Danilova, 1979; Diogo and Wood, 2012].

In all dissected platyrrhines, $\mathrm{m}$. extensor digitorum lateralis typically controls digits IV-V, which is consistent with the published data. Although 2 heads, bearing separate tendons, are more common, the variant, with an initially single tendon bifurcating distally, found in Cebuella pygmaea, is also known for Callimico, Callithrix and Saguinus [Hill, 1959]. However, Hill [1957] observed only 1 tendon to the fourth

${ }^{1}$ Fourteen specimens were examined. 
finger in Callimico; likewise there are descriptions of a single tendon to the fifth finger in Callithrix [Gremyatskiy, 1933] and Cebus [Hill, 1960].

The most significant variant of this muscle was found in Callicebus [Bodini, 1985]. In addition to the typical head sending a bifurcating tendon to digits IV and $\mathrm{V}$, there was an extra separate head supplying digit $\mathrm{V}$, which originated at the midlength of the ulna.

In cercopithecids as a rule, $\mathrm{m}$. extensor digitorum lateralis also operates 2 fingers $(I V-V)$. The following pair of cases described in the literature can be treated as anomalies: control of only digit IV in Papio hamadryas [Hill, 1970, as cited in Diogo and Wood, 2012], and control of only digit V in 1 left forelimb of Colobus guereza [Jouffroy, 1962]. In gibbons, $\mathrm{m}$. extensor digitorum lateralis operates only the fifth finger. The great apes demonstrate a great range of variations: Pongo IV-V (more common) or V; Gorilla IV, IV-V (more common), or V; Pan IV-V or V (more common) [Sonntag, 1923; Diogo and Wood, 2012]. In humans, m. extensor digitorum lateralis normally operates exclusively the fifth finger, but abnormal variants with 2 tendons going to fingers IV-V are known [Kaneff and Čihák, 1970; Danilova, 1979; Sinelnikov, 1989].

\section{Discussion}

As it is evident from the results, the most variable muscle of the deep extensor complex is $\mathrm{m}$. extensor digiti secundi. Its tendon distribution looks quite diverse and random, at first glance. Of course, it cannot be excluded that researchers could, in some cases, have encountered individuals abnormal in tendon architecture, which makes it difficult to evaluate a morphotype characteristic for certain species. But unfortunately there are no studies focusing on individual variability of m. extensor digiti secundi in platyrrhines and in primates in general, except humans.

In our opinion, the extensive list of tendon branching variants in $\mathrm{m}$. extensor digiti secundi could have arisen partially due to the absence of a general consensus on the identification and description of the terminal tendons: the question is whether we are dealing with proper terminal tendons, or just hardenings of the connecting aponeurosis. When an author describes in detail the location of tendon branching or provides detailed illustrations, verification is not a problem; otherwise, we need to rely on the author's conclusions and accept the proposed point of view. Moreover, tendons of $\mathrm{m}$. extensor digiti secundi may be connected with each other by a more or less strong aponeurosis, the sides of which are attached to metacarpals I and V. Occasionally the connective tissue of this aponeurosis locally thickens and becomes similar to tendons; according to Diogo and Wood [2012]; such formations can be regarded as additional tendons of $\mathrm{m}$. extensor digiti secundi. Perhaps this might be the case for the tendon of $\mathrm{m}$. extensor digiti secundi to digit $\mathrm{V}$ in tarsiers [Straus, 1941; Danilova, 1979; Diogo and Wood, 2012], as well as to digit IV in Lemur, Tarsius, and Hylobates [Straus, 1941]. Diogo and Wood [2012] argued that this pattern could have been observed by many other researchers, but was interpreted as $\mathrm{m}$. extensor digiti secundi II-III as a result of ignoring any minor aponeurotic thickenings. We have observed something similar in some of our dissections. Most commonly it was a connective tissue membrane with multi-directional fibres, thin and fragile, unlike a proper tendon transmitting the muscular force. Such a membrane should be considered

Extensor Digitorum Profundus Muscle Complex in Platyrrhini
Folia Primatol 2017;88:274-292 DOI: $10.1159 / 000478524$
285 
an indicator of mutual immobility of proper tendons, but it cannot carry any functional load, as it tears easily and is unable to transmit the muscular force. In marmosets, parts of this membrane can easily be mistaken for additional tendinous branches to the lateral fingers. For that reason, variants II-IV, II-V, II and IV, II and V, III-IV, III and V, referred to but not described in detail in the literature, seem doubtful.

On the whole, variations in the structure of the muscles under consideration can be divided into 2 main types. In the first type, the terminal tendons are initially separate, i.e., each serves its own muscle fibres, and the muscle in fact has independent heads, which can separately control corresponding fingers. The number of heads of $\mathrm{m}$. extensor digiti secundi can vary from 1 to 3 ; in m. extensor pollicis longus and $\mathrm{m}$. extensor digitorum lateralis there can be 1 or 2 heads. In the second structural type, all fibres of the muscle pull on a single terminal tendon, which then divides into several branches to the digits. With such a branching terminal tendon, the muscle jointly operates several fingers (up to 4).

Youlatos [1999] argued that the differentiation of the deep digital extensors and the distribution of their tendons are functionally associated with the thumb opposition in humans versus schizodactylous grasp (an object is placed between digits II and III) of the howler monkey. On the one hand, he considered humans capable of opposing the thumb, in which every finger tendon has its own muscle belly. On the other hand, in Alouatta, which is able to spread apart the second and third fingers, the tendons passing to the first and second fingers represent branches of one common tendon of $\mathrm{m}$. extensor pollicis longus, and there is a separate muscle for the third finger (it is the $\mathrm{m}$. extensor digiti secundi) ${ }^{2}$.

There is no doubt that the great manipulatory abilities of humans determine a high degree of differentiation in muscles serving the manus. However, our data demonstrate that most of the variations in tendons cannot be explained by differences in manipulatory skills. For example, marmosets use "squirrel-like" locomotion (i.e., clawed quadrupedal running and jumping on the trunks and branches of trees), where they spread their fingers apart but do not use separate extension of any particular finger. Additionally, when manipulating objects, marmosets also tend to use the simplest grasp: they press the item to their palm with 4 fingers [Deryagina, 1986]. However, it is in marmosets that we observe the greatest diversity in the distribution of terminal tendons of $\mathrm{m}$. extensor digiti secundi and m.extensor pollicis longus: $\mathrm{Ce}$ buella pygmaea ( $\mathrm{m}$. extensor pollicis longus to fingers I-III, $\mathrm{m}$. extensor digiti secundi to III-IV), Callithrix jacchus (m. extensor pollicis longus to I-II, m. extensor digiti secundi to III-IV), Leontopithecus rosalia (m. extensor pollicis longus to I-II, $\mathrm{m}$. extensor digiti secundi to II-III). What is not well developed in marmosets is the differentiation of muscular heads supplied with individual tendons.

On this basis, we can put forward the suggestion that maintenance of the high potential for manipulation is associated with the formation of independent muscle heads, 1 tendon per head, while the multiple branching of tendons of a single muscle head, on the contrary, is typical for animals with undifferentiated control of their fingers (whole-arm control sensu Bishop [1962]). A similar argument was suggested by Abdala et al. [2009] on the degrees of tendon separation in lizards, although not in extensors but in the long digital flexor. The functional principle is analogous. How-

${ }^{2}$ In humans, the system of deep extensors does not supply the third finger at all. 


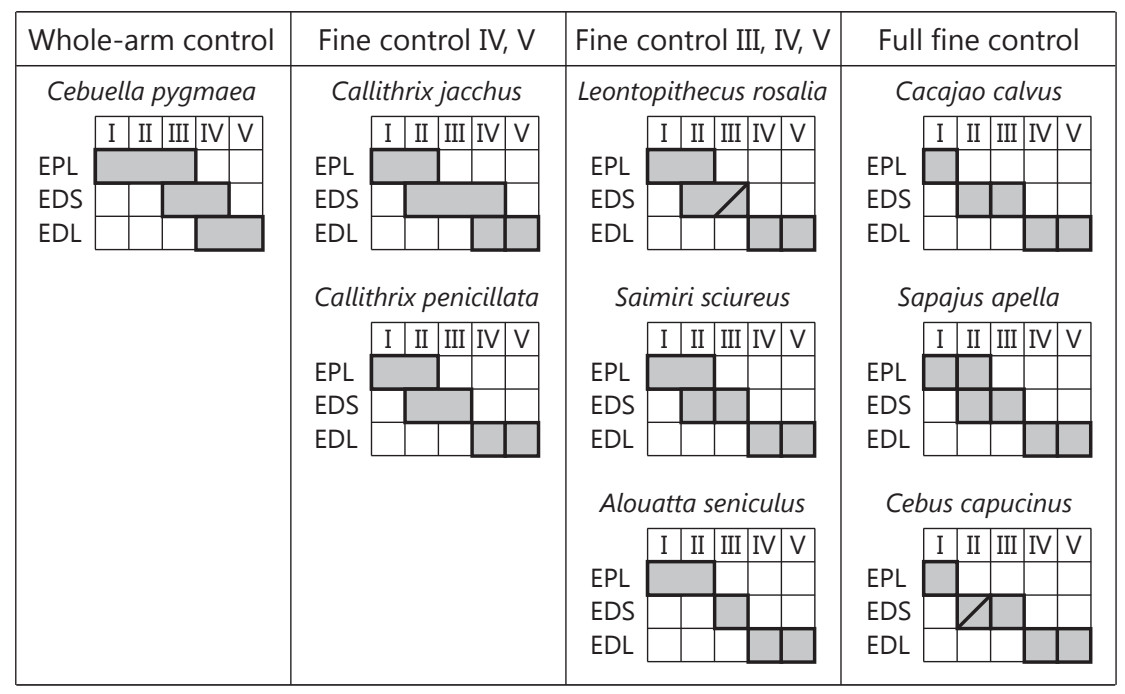

Fig. 2. The summary of associations of the deep extensor muscles with the digits in the studied platyrrhines. The diagrams are simplified from Table 2 and ordered from the left to the right according to the degree of separation of tendons to individual fingers. EPL, m. extensor pollicis longus; EDS, m.extensor digiti secundi; EDL, m. extensor digitorum lateralis.

ever, there is an important difference, as flexors are responsible for prehensive grip, while extensors produce a preparatory prehensive pattern sensu Bishop [1962].

Based on our data on the structure of deep extensors of fingers, several morphofunctional gradations can be distinguished in platyrrhines with respect to precision of the prehensive pattern (Fig. 2). The whole-arm control, characterized by the absence of an individual extensor of any single digit, is specific to Cebuella. Callithrix shows the structural basis for separate control of the lateral digits (IV, V), but extension of digits I-III is coupled in one way or the other. Indeed, marmosets do not use their hands for precise manipulation. Squirrel monkeys and howler monkeys have gained the morphological separation of the extensor muscle of digit III, so only the control of digits I and II remains coupled. A similar condition in Leontopithecus functionally contradicts its peculiar syndactyly II-IV and may be regarded as the remnant of an ancestral, nonsyndactylous, state. Finally, in uakaris and capuchins, the structural basis for complete fine control over every finger individually is achieved through entire separation of the heads of the deep extensor muscle complex. Uakaris and capuchins belong to 2 different platyrrhine lineages (families), but both are known for their elaborate manipulatory abilities [Bishop, 1964; Parker and Gibson, 1977; Deryagina, 1997]. In general, there is an obvious mediolateral polarity in the order of finger coupling or discoupling among platyrrhines. Finger separation progresses from the lateral to medial fingers, and finger coupling goes in the opposite direction. Apparently, this rule is true irrespective of phylogenetic positions and relations of the species studied.

As plesiomorphic state for primates, Diogo and Wood [2012] considered the presence of tendons II-III in $\mathrm{m}$. extensor digiti secundi. This morphology occurs in 
most cases in strepsirrhines, tarsiers and cercopithecids, in about half of the cases in platyrrhines, rarely in hominoids (inter alia in about half of orang-utan specimens), and as an anomaly in humans. However, the presence of the variants II and II-III in tree shrews [Le Gros Clark, 1924; Panyutina et al, 2015] provides evidence in favour of high variability of this structural characteristic within Euarchonta in general ${ }^{3}$. In the opinion of Dunlap et al. [1985], based on data from Straus [1941], for all eutherian mammals the primitive distribution of tendons of $\mathrm{m}$. extensor digiti secundi is digits II-IV. That is why it would be premature to draw any conclusions concerning the plesiomorphic state for primates, on the basis of their own diversity alone.

The study of the earlier evolution of this muscle complex composition could help understand the sources of its current diversity. It is well established that initially all the deep extensor heads were short (i.e., originated on carpal bones, like the heads of the short extensors of the foot which originate on the tarsals) [Diogo and Abdala, 2010]. From this starting point, the following scenario of their proximal displacement to the forearm and humerus might be proposed. Most likely, initially there were 5 muscle heads, one for each finger, as in reptiles, the tendons of which reach the ungual phalanges, while $\mathrm{m}$. extensor digitorum communis terminates on the metacarpals in sharp contrast with the mammalian condition [Miner, 1925; Haines, 1939]. We can assume that during the reptile-mammal transition all 5 heads began to shift proximally (like the heads of the deep flexor of the digits). Extant monotremes show a peculiar mixture of primitive, derived and specific features [Gambaryan et al., 2015]. The extensor digitorum profundus complex is divided into 2 muscles - the $\mathrm{m}$. extensor digitorum lateralis and the single $\mathrm{m}$. extensor digitorum medialis (i.e., pollicis et indicis together). The tendon of the first one bifurcates and supplies digits IV and V, and the tendon branches of the second one supply digits I-III in Ornithorhynchus, I-IV in Tachyglossus, and II-IV in Zaglossus (branch I is absent due to thumb reduction). A primitive, reptilian-level feature is the insertion of all these branches on the ungual phalanges (only branch $\mathrm{V}$ in Zaglossus is inserted on the pre-ungual phalanx due to reduction of the ungual one); so there is double control of the ungual phalanges by the deep extensor complex (reptilian control) and by the superficial $\mathrm{m}$. extensor digitorum communis (therian control). As to the proximal shift of the muscular origin, the m. extensor digitorum lateralis has reached the proximal aponeurosis of the m. extensor digitorum communis (and, in Zaglossus, it has even spread over it onto the humeral ectepicondylus). The m. extensor digitorum medialis has shifted proximally onto the olecranon in Ornithorhynchus, but in tachyglossids it shows a more primitive condition originating along the ulnar shaft but not from the olecranon. Sometimes vivid traces of this proximal shift are retained in more advanced mammals than monotremes, such as the aforementioned extra head of m. extensor digitorum lateralis in Callicebus, with an origin restricted to the distal half of the ulna [Bodini, 1981]; another example is the human anomaly of the extensor digiti secundi with its origin displaced downwards up to carpal bones [Straus, 1941; Aziz and Dunlap, 1986]. Short heads of deep extensors are also known as an anomaly in Ateles [Straus, 1941] and as frequent variations in Bradypus, Choloepus, Cyclopes, and Manis [Humphry, 1870; Windle and Parsons, 1899; Mendel, 1981]. In the course of proximal displacement, the heads of the deep extensor were

\footnotetext{
${ }^{3}$ In Colugo this muscle is arranged in a very peculiar manner and bears tendons to digits II-IV [Panyutina et al., 2015].
} 
combined in 2 groups: the medial (supplying digits I, II, and III) and the lateral (supplying digits IV and V) ones. This grouping is exemplified by some tree shrews, in which extensor heads to digits I-III were even termed jointly as " $m$. extensor digitorum radialis" and heads to digits IV-V as " $\mathrm{m}$. extensor digitorum ulnaris" [George, 1977]. The assumption that the head to the third digit joined the medial group is supported by the fact that the bundle of muscle fibres serving the tendon of this digit is found in mammals almost always as a part of $\mathrm{m}$. extensor digiti secundi. If digit III is operated by $\mathrm{m}$. extensor digitorum lateralis, its tendon represents an offshoot of the tendon to digit IV or a common tendon to digits IV and V, before its bifurcation, and it does not have its own muscle fibres. So this is not a true separate head in the full sense in this case. The disappearance of the head for the third digit, which often occurs in primates (Callithrix jacchus, Papio, Homo) and in many other placentals as well (e.g., Tupaia belangeri), could be due to the fact that $\mathrm{m}$. extensor digitorum communis in basal mammals took control over the ends of digits by transferring its insertions to the distal phalanges. Double supply of the ungual phalanges by the deep extensor complex together with $\mathrm{m}$. extensor digitorum communis can be observed already in monotremes [Gambaryan et al., 2015]. The further process is well illustrated in opossums: the double supply of the ungual phalanges is retained, but $\mathrm{m}$. extensor digitorum communis has lost the tendon to the thumb, while the deep extensor complex has lost the head for digit III [Stein, 1981]. Thus, after the $\mathrm{m}$. extensor digitorum communis had taken over the control of clawed phalanges from the deep extensor complex, the number of heads of the latter began to vary depending on manual adaptations to manipulation. When necessary, a full set of heads can be restored or even multiplied for fine control over digits (e.g., Cebus, Cacajao). The opposite tendency towards integral control of several digits by the same muscular head is achieved through tendon branching (e.g., Cynocephalus, Cebuella). Only in the thumb, the deep extensor retains exclusive control over the clawed phalanx, and this finger is not supplied by $\mathrm{m}$. extensor digitorum communis in extant therians. This is not surprising, as the first finger in mammals has 1 phalanx less and does not need a double system for controlling extension.

\section{Conclusions}

Here we summarize the suggested transformations of the digital extensors.

1 Premammalian condition (found in reptiles). M. extensor digitorum communis reaches from the forearm to the metacarpals. The extensor digitorum profundus complex consists of 5 short heads connecting the wrist base with the ungual phalanges.

2 Basal mammalian condition (found in monotremes). M. extensor digitorum communis transfers its terminal tendon branches distally, and the double control of the ungual phalanges of all digits arises. The extensor digitorum profundus complex expands proximally over the forearm and is divided into 2 bundles - the medial heads (I-III) and the lateral ones (IV, V); the terminal tendons in each bundle may be united proximally.

3 Basal therian condition. M. extensor digitorum communis loses the tendon to digit I.

Extensor Digitorum Profundus Muscle Complex in Platyrrhini
Folia Primatol 2017;88:274-292 DOI: $10.1159 / 000478524$
289 
4 Basal placental condition. The tendons of the extensor digitorum profundus complex are transferred proximally, to the pre-ungual phalanges of digits II-V. So, the distal joints of these digits are controlled singly by $\mathrm{m}$. extensor digitorum communis.

5 Primate condition. The tendons of the extensor digitorum profundus complex fuse into those of the $\mathrm{m}$. extensor digitorum communis to form a common tendinous sheath reaching the nail base. So, double control of the ungual phalanges of digits II-V is restored. If the tendons of the extensor digitorum profundus complex are separate from each other from the very beginning, its heads (they may be even multiplied) make fine control of digits for a preparatory prehensive pattern. If the tendons are fused proximally as branches of a common stem, the respective heads can only help m. extensor digitorum communis in whole-arm control.

\section{Acknowledgments}

We are very grateful to Mr. Eugenii L. Yakhontov, who helped us with the English translation and with the literature. We also acknowledge the valuable advice of Alexander N. Kuznetsov. 05049-a).

The work was supported by the Russian Foundation for Basic Research (grant No. 15-04-

\section{Disclosure Statement}

No conflict of interest exists.

\section{References}

Abdala V, Manzano AS, Tulli MJ, Herrel A (2009). The tendinous patterns in the palmar surface of the lizard manus: functional consequences for grasping ability. The Anatomical Record 292: 842-853.

Ankel-Simons F (2007). Primate Anatomy: An Introduction. Durham, Academic Press.

Aversi-Ferreira TA, Diogo R, Potau JM, Bello G, Pastor JF, Aziz MA (2010). Comparative anatomical study of the forearm extensor muscles of Cebus libidinosus (Rylands et al., 2000; Primates, Cebidae), modern humans, and other primates, with comments on primate evolution, phylogeny, and manipulatory behavior. The Anatomical Record 293: 2056-2070.

Aziz MA, Dunlap SS (1986). The human extensor digitorum profundus muscle with comments on the evolution of the primate hand. Primates 27: 293-319.

Barnard WS (1876). Observations on the membral musculation of Simia satyrus (Orang) and the comparative myology of man and the apes. Proceedings of the American Association for the Advancement of Science 24: 112-144.

Beattie J (1927). The anatomy of the common marmoset (Hapale jacchus Kuhl). Proceedings of the Zoological Society of London 97: 593-718.

Bishop A (1962). Control of the hand in lower primates. Annals of the New York Academy of Sciences 102: 316-337.

Bishop A (1964). Use of the hand in lower primates. In Evolutionary and Genetic Biology of Primates (Buettner-Janusch J, ed.), pp 133-223. New York, Academic Press.

Bodini R (1981). Musculatura locomotora de la viudita (Callicebus torquatus). Sus implicaciones funcionales y filogenéticas. Memoria de la Sociedad de Ciencias Naturales La Salle 116: 9-163.

Burmeister H (1846). Beiträge zur näheren Kenntnis der Gattung Tarsius. Berlin, Reimer.

Čihák R (1991). Phylogenetically ancient pattern in ontogenesis of limb muscles. In Developmental Patterning of the Vertebrate Limb (Hinchliffe JR, Hurle JM, Summerbell D, eds.), pp 355-363. New York, Springer.

Danilova EI (1979). Evolution of the Hand (in Russian). Kiev, Vysha Shkola. 
Deniker J (1885). Recherches anatomiques et embryologiques sur les singes anthropoides. Foetus de gorille et de gibbon. Archives de zoologie expérimentale et générale 3: 1-265.

Deryagina MA (1986). Manipulatory Activity of Primates (Ethological Analysis in Connection with the Problems of Anthropogenesis) (in Russian). Moscow, Academy of Sciences Publishing.

Deryagina MA (1997). Evolution of the Primate Behavior: The Ethological Approach to the Problems of Anthroposociogenesis. Doctor of Science dissertation, Lomonosov Moscow State University.

Diogo R, Abdala V (2010). Muscles of Vertebrates - Comparative Anatomy, Evolution, Homologies and Development. Enfield, Science Publishers.

Diogo R, Wood BA (2012). Comparative Anatomy and Phylogeny of Primate Muscles and Human Evolution. Jersey, Science Publishers.

Diogo R, Potau JM, Pastor JF, de Paz FJ, Ferrero EM, Bello G, Barbosa M, Wood BA (2010). Photographic and Descriptive Musculoskeletal Atlas of Gorilla: With Notes on the Attachments, Variations, Innervation, Synonymy and Weight of the Muscles. Enfield, CRC Press.

Duckworth WLH (1904). Studies from the Anthropological Laboratory, the Anatomy School. Cambridge, Cambridge University Press.

Dunlap SS, Thorington RW, Aziz MA (1985). Forelimb anatomy of New World monkeys: myology and the interpretation of primitive anthropoid models. American Journal of Physical Anthropology 68: 499-517.

Gambaryan PP, Kuznetsov AN, Panyutina AA, Gerasimov SV (2015). Shoulder girdle and forelimb myology of extant Monotremata. Russian Journal of Theriology 13: 1-56.

George RM (1977). The limb musculature of the Tupaiidae. Primates 18:1-34.

Grand T (1968). Functional anatomy of the upper limb. In Biology of the Howler Monkey (Alouatta caraya) (Malinow MR, ed.), Bibliotheca Primatologica No 7, pp 104-125. Basel, Karger.

Gremyatskiy MA (1933). Neuromuscular system of the forelimb of Hapale jacchus Kuhl (in Russian). Antropologicheskiy Zhurnal 1-2: 201-215.

Haines RW (1939). A revision of the extensor muscles of the forearm in tetrapods. Journal of Anatomy 73: 211-233.

Hershkovitz P (1977). Living New World Monkeys (Platyrrhini). Chicago, University of Chicago Press.

Hill WCO (1953). Primates - Comparative Anatomy and Taxonomy. I. Strepsirhini. Edinburgh, Edinburgh University Press.

Hill WCO (1957). Primates - Comparative Anatomy and Taxonomy. III. Pithecoidea, Platyrrhini (Families Hapalidae and Callimiconidae). Edinburgh, Edinburgh University Press.

Hill WCO (1959). The anatomy of Callimico goeldii (Thomas): a primitive American primate. Transactions of the American Philosophical Society 49: 1-116.

Hill WCO (1960). Primates - Comparative Anatomy and Taxonomy. IV. Cebidae, Part A. Edinburgh, Edinburgh University Press.

Hill WCO (1962). Primates - Comparative Anatomy and Taxonomy. V. Cebidae, Part B. Edinburgh, Edinburgh University Press.

Hill WCO (1970). Primates - Comparative Anatomy and Taxonomy. VIII. Cynopithecinae: Papio, Mandrillus, Theropithecus. Edinburgh, Edinburgh University Press.

Humphry GM (1870). The myology of the limbs of the unau, the ai, the two-toed anteater, and the pangolin. Journal of Anatomy and Physiology 4: 17-78.

International Committee on Veterinary Gross Anatomical Nomenclature (2012). Nomina anatomica veterinaria, 5 th ed. World Association of Veterinary Anatomists.

Jouffroy FK (1962). La musculature des membres chez les Lémuriens de Madagascar: étude descriptive et comparative. Mammalia 26: 1-324.

Jouffroy FK, Lessertisseur J (1960). Les spécialisations anatomiques de la main chez les singes à progression suspendue. Mammalia 24: 93-151.

Kaneff A (1980). Évolution morphologique des musculi extensores digitorum et abductor pollicis longus chez l'homme. III. Évolution morphologique du m. extensor indicis chez l'homme, conclusion générale sur l'évolution morphologique des musculi extensores digitorum et abductor pollicis longus chez l'homme. Gegenbaurs Morphologisches Jahrbuch 126: 774-815.

Kaneff A, Čihák R (1970). Die Umbildung des M. extensor digitorum lateralis in der Phylogenese und in der menschlichen Ontogenese. Acta Anatomica 77: 583-604.

Le Gros Clark WE (1924). The myology of the tree-shrew (Tupaia minor). Proceedings of the Zoological Society of London 94: 461-497.

Mendel FC (1981). The hand of two-toed sloths (Choloepus): its anatomy and potential uses relative to size of support. Journal of Morphology 169: 1-19.

Miner RW (1925). The pectoral limb of Eryops and other primitive tetrapods. Bulletin of the American Museum of Natural History 51: 1875-1955.

Murie J, Mivart G (1872). On the anatomy of the Lemuroidea. Transactions of the Zoological Society of London 7: 1-113.

Napier JR (1961). Prehensility and opposability in the hands of primates. Symposia of the Zoological Society of London 5: 115-132.

Extensor Digitorum Profundus Muscle Complex in Platyrrhini
Folia Primatol 2017;88:274-292 DOI: $10.1159 / 000478524$
291 
Novikova MA, Kuznetsov AN (2017). An unusual elbow muscle in the red howler monkey: does it deserve invention of a new name musculus contrahens cubiti? Biological Communications 62: 33-49.

Panyutina AA, Korzun LP, Kuznetsov AN (2015). Flight of Mammals: From Terrestrial Limbs to Wings. Cham, Springer.

Parker ST, Gibson KR (1977). Object manipulation, tool use and sensory motor intelligence as feeding adaptations in Cebus monkeys and great apes. Journal of Human Evolution 6: 623-641.

Perelman P, Johnson WE, Roos C, Seuánez HN, Horvath JE, et al (2011). A molecular phylogeny of living primates. PLoS Genetics 7: e1001342.

Pocock RI (1917). The genera of Hapalidae (marmosets). Annals and Magazine of Natural History 20: $247-258$.

Raven HC (1950). Regional anatomy of the gorilla. In The Anatomy of the Gorilla (Gregory WK, ed.), pp 15-188. New York, Columbia University Press.

Robertson DF (1944). Anatomy of the South American woolly monkey (Lagothrix). 1. The forelimb. Zoologica 29: 169-192.

Schneider H, Sampaio I (2015). The systematics and evolution of New World primates - a review. Molecular Phylogenetics and Evolution 82: 348-357.

Schön MA (1968). The muscular system of the red howling monkey. Bulletin of the United States National Museum 273: 1-185.

Sinelnikov RD (1989). Atlas of Human Anatomy. Vol 1: Musculoskeletal System. Moscow, Mir Publisher.

Soligo C (2005). Anatomy of the hand and arm in Daubentonia madagascariensis: a functional and phylogenetic outlook. Folia Primatologica 76: 262-300.

Sonntag CF (1923). On the anatomy, physiology, and pathology of the chimpanzee. Proceedings of the Zoological Society of London 93: 323-429.

Springer MS, Meredith RW, Gatesy J, Emerling CA, Park J, et al (2012). Macroevolutionary dynamics and historical biogeography of primate diversification inferred from a species supermatrix. PLoS One 7: e49521.

Stein BR (1981). Comparative limb myology of two opossums, Didelphis and Chironectes. Journal of Morphology 169: 113-140.

Stevens JL, Meyer DM, Edgerton VR (1977). Gross anatomy of the forelimb and shoulder girdle of the Galago senegalensis. Primates 18: 435-452.

Straus WL (1941). The phylogeny of the human forearm extensors. Human Biology 13: 23-50.

Sullivan WE, Osgood CW (1927). The musculature of the superior extremity of the orangutan, Simia satyrus. The Anatomical Record 35: 193-239.

Sustaita D, Pouydebat E, Manzano A, Abdala V, Hertel F, Herrel A (2013). Getting a grip on tetrapod grasping: form, function, and evolution. Biological Reviews 88: 380-405.

Turnquist JE (1983). Forelimb musculature and ligaments in Ateles, the spider monkey. American Journal of Physical Anthropology 62: 209-226.

Windle BCA, Parsons FG (1899). On the myology of the Edentata. Proceedings of the Zoological Society of London 67: 314-339.

Woollard HH (1925). The anatomy of Tarsius spectrum. Proceedings of the Zoological Society of London 70: $1071-1184$.

Youlatos D (1999). The schizodactylous grasp of the howling monkey. Zeitschrift für Morphologie und Anthropologie 82: 187-198.

Youlatos D (2000). Functional anatomy of forelimb muscles in Guianan Atelines (Platyrrhini: Primates). Annales des Sciences Naturelles - Zoologie et Biologie Animale 21: 137-151.

Ziemer LK (1972). An Atlas of the Skeleton and Musculature of the Shoulder, Arm and Forearm of Pithecia monacha. MA thesis, Johns Hopkins University, Baltimore. 